\title{
Modelling of heat and mass transfer processes in neonatology
}

\author{
Maciej K. Ginalski ${ }^{1}$, Andrzej J. Nowak ${ }^{2}$ and Luiz C. Wrobel ${ }^{3}$ \\ ${ }^{1}$ FLUENT Europe, Sheffield Business Park, Europa Link, Sheffield S9 1XU, United \\ Kingdom \\ ${ }^{2}$ Institute of Thermal Technology, Silesian University of Technology, Konarskiego \\ 22, 44-100 Gliwice, Poland \\ ${ }^{3}$ School of Engineering and Design, Brunel University, Uxbridge UB8 3PH, United \\ Kingdom
}

E-mail: $\quad$ maciej.ginalski@ansys.com, Andrzej.J.Nowak@polsl.pl, luiz.wrobel@brunel.ac.uk

This paper reviews some of our recent applications of Computational Fluid Dynamics (CFD) to model heat and mass transfer problems in neonatology and investigates the major heat and mass transfer mechanisms taking place in medical devices such as incubators and oxygen hoods. This includes novel mathematical developments giving rise to a supplementary model, entitled Infant Heat Balance Module, which has been fully integrated with the CFD solver and its graphical interface. The numerical simulations are validated through comparison tests with experimental results from the medical literature. It is shown that CFD simulations are very flexible tools that can take into account all modes of heat transfer in assisting neonatal care and the improved design of medical devices.

Keywords: Computational fluid dynamics, heat and mass transfer, neonatology

\section{Introduction}

The maintenance of an optimal thermal environment is regarded as a priority in neonatology. While healthy neonates are able to regulate their body temperature, premature and small infants may have difficulties adapting to temperature changes. For this reason, keeping an optimum environmental temperature is essential for their healthy development and even survival.

Computational Fluid Dynamics (CFD) has been greatly developed over recent years, mostly due to the rapid advance of computer technology. It is now possible to simulate scientific problems with complex geometries, including several combined processes taking place simultaneously. This paper reviews some of our recent applications of CFD to problems in neonatology and investigates the major mechanisms of heat transfer taking place in medical devices such as incubators and oxygen hoods.

Numerical techniques have been successfully used to describe the thermal interaction between the human body and its surrounding environment [1-4]. However, most of these studies were undertaken with reference to adults whose physical shape, thermo-physiological 
properties and thermoregulatory processes are different from those of infants [5-7]. Studies investigating physical processes occurring inside closed environments such as incubators have mostly been carried out through experimental techniques [8-11]. Numerical simulations are very useful in many situations as they easily allow parametric testing, can provide a wealth of data and assist in the design of more efficient, safe and reliable medical equipment.

The present paper analyses the heat and mass transfer mechanisms of metabolic heat generation and sensible and latent heat losses, including heat conduction, convection, radiation and evaporation. The paper follows previous studies by Ginalski et al. [12, 13], who presented a model for analysing the total heat balance of the neonate using CFD techniques. Moreover, significant improvements have been made to the geometric representation of the infants and the process of data generation. These developments gave rise to a supplementary model, entitled Infant Heat Balance Module (IHBM), which has been fully integrated with the CFD solver and graphical interface. The current version of the model is capable of calculating all the heat balance components that are present during the intensive care of neonates under different treatment scenarios.

\section{Heat Transfer from the Human Body}

The metabolic heat generated in the body is dissipated to the environment as sensible heat through the skin and lungs, and as latent heat by evaporation. Sensible heat loss from the skin depends on the temperatures of the skin, the environment, and the surrounding surfaces as well as the air motion. Latent heat loss, on the other hand, represents the heat of vaporization of water as it evaporates in the lungs and on the skin by absorbing body heat, and is released as the moisture condenses on cold surfaces. It also depends on the skin wettedness and the relative humidity of the environment.

The total rate of heat loss from the body can be expressed as [14]:

$$
\left(\dot{Q}_{m e t}+\dot{Q}_{b l}\right)_{g}=\left(\dot{Q}_{c d}+\dot{Q}_{c v}+\dot{Q}_{r}+\dot{Q}_{e v}\right)_{s k}+\left(\dot{Q}_{c v}+\dot{Q}_{e v}\right)_{l u}
$$

where:

$\left(\dot{Q}_{m e t}\right)_{g}$ - rate of heat generated by metabolism

$\left(\dot{Q}_{b l}\right)_{g}$ - rate of heat dissipated by blood perfusion

$\left(\dot{Q}_{c d}\right)_{s k}$ - rate of heat loss from the skin due to conduction

$\left(\dot{Q}_{c v}\right)_{s k}$ - rate of heat loss from the skin due to convection

$\left(\dot{Q}_{r}\right)_{s k}$ - rate of heat loss from the skin due to radiation

$\left(\dot{Q}_{e v}\right)_{s k}$ - rate of latent heat of evaporation from the skin

$\left(\dot{Q}_{c v}\right)_{l u}$ - rate of heat loss by convection through respiration

$\left(\dot{Q}_{e v}\right)_{l u}$ - rate of latent heat by evaporation through respiration

Under study conditions, the total rate of heat transfer from the adult body is equal to the rate of metabolic heat generation in the body, which varies from about $100 \mathrm{~W}$ for light office work to roughly $1000 \mathrm{~W}$ during heavy physical work [14]. In the case of infants, right after birth, this value is very small and usually does not exceed 5W [11]. 


\section{Infant Heat Balance Module}

The governing equations of fluid flow and associated phenomena have been solved in the present study with the utilization of the commercial CFD solver FLUENT [15]. Heat exchange by conduction, convection and radiation between the neonate and the surrounding environment is determined through the solution of the mass, momentum and energy conservation equations. The Boussinesq approximation was adopted for the buoyancy term in the momentum conservation equation.

The temperature on the surface of the mattress on which the infant lies is set to be equal to the infant's skin temperature. Therefore, no conduction heat loss between infant and mattress was considered in this study. This is a reasonable assumption following Wheldon [16], who states that the rate of heat transfer by conduction is small for a baby lying on a foam mattress. The airflow is considered to be turbulent and viscous. The Shear-Stress Transport (SST) $k-\omega$ turbulence model has been used in all simulations, with a correction for flows characterized by a low Reynolds number. Radiation heat exchange is calculated in the FLUENT solver using the discrete ordinates radiation model. Air was considered to be a transparent medium for radiation modelling. Detailed information is provided in [12] and [15].

The standard version of the solver possesses several limitations that reduce its applicability to calculate processes crucial for analysing the thermal balance of neonates. Some of the components of the infant heat balance described in Equation (1), including heat generation and dissipation within the infant's body, heat loss due to evaporation from the skin and the respiratory tract, heat loss due to conduction and convection in the lungs, cannot be solved by the standard version of FLUENT. Hence, a series of developments were necessary to allow the calculation of all the components of the infant heat balance in Equation (1). A supplementary module, entitled Infant Heat Balance Module (IHBM), was then developed and fully integrated in the FLUENT main solver and graphical interface.

Most of the mathematical analysis carried out in bioheat transfer to date is based on Pennes' equation [17]. Capabilities for solution of the bioheat equation are also available in the FLUENT solver. Metabolic contributions are included as distributed energy sources. Other effects also included as heat sources in the bioheat equation are the heat transferred with blood per unit volume of tissue, moisture evaporation from the infant's skin and latent heat of evaporation from the infant's skin per unit volume of tissue.

An energy balance for a control volume of tissue with heat sources can be formulated as follows [17]:

$$
\frac{\partial T_{t s}}{\partial t} \rho_{t s} C_{t s}=\lambda_{t s} \nabla^{2} T_{t s}+\dot{q}_{t o t}
$$

where $T_{t s}, \lambda_{t s}, \rho_{t s}$ and $C_{t s}$ are the tissue temperature, thermal conductivity, density and specific heat, respectively, $t$ is time and the source term can be defined in the form:

$$
\dot{q}_{t o t}=\dot{q}_{m e t}+\dot{q}_{b l}+\dot{q}_{e v}+\dot{q}_{l u}
$$

where:

$$
\begin{aligned}
& \dot{q}_{m e t} \text { - rate of metabolic heat generated per unit volume of tissue } \\
& \dot{q}_{b l} \text { - rate of heat transferred with blood per unit volume of tissue } \\
& \dot{q}_{e v} \text { - rate of evaporation heat loss from the infant's skin per unit volume of tissue } \\
& \dot{q}_{l u} \text { - rate of respiration heat loss per unit volume of tissue }
\end{aligned}
$$


Empirical approximations for modelling the above terms are discussed below. These approximations have been implemented in the IHBM module to supplement the FLUENT solver.

\subsection{Modelling blood heat generation}

The blood acts as a local distributed, scalar source or sink of energy with magnitude equal to:

$$
\dot{q}_{b l}=\zeta \rho_{b l} C_{b l}(1-\kappa)\left(T_{c r}-T_{t s}\right)
$$

where $\zeta, \rho_{b l}$ and $C_{b l}$ are the blood perfusion rate, density and specific heat, respectively; $\kappa$ is a factor accounting for the incomplete thermal equilibrium between blood and tissue; and $T_{c r}$ is the arterial blood temperature.

\subsection{Modelling metabolic heat generation}

The total metabolic heat transfer generated by the neonate is calculated based on an empirical formula introduced by Brück [18]:

$$
\dot{q}_{m e t}=\frac{m_{\mathrm{inf}}\left(0.0522 \tau_{\mathrm{inf}}+1.64\right)}{V_{\mathrm{inf}}}
$$

where $m_{\text {inf }}$ is the infant's mass, $\tau_{\text {inf }}$ is the infant's age (in days) and $V_{\text {inf }}$ is the volume of the infant's body.

\subsection{Modelling latent heat of evaporation}

Assume, initially, that the water temperature at the infant's skin and the temperature of the surrounding air are the same. If the air is saturated, there will be no heat or mass transfer while the isothermal conditions remain unchanged. On the other hand, if the air is not saturated, there will be a difference between the water vapour concentration at the skin and the surrounding air, which will drive the mass transfer process. However, before mass transfer can occur, water must first evaporate. The evaporation process requires that heat be absorbed by the evaporating liquid. Initially, this heat will come from the skin. The temperature of a thin layer of water at the skin surface must drop as a result of sensible heat loss, which also reduces the saturation pressure and thus the vapour concentration at the interface. This temperature drop creates temperature differences within the skin, as well as between the skin and the surrounding air. These temperature differences drive heat transfer toward the skin surface from both the air and the deeper parts of the body.

If the evaporation process is high and, therefore, the demand for the heat of evaporation is higher than the amount of heat that can be supplied by the lower parts of the skin and the surroundings, the deficit will be made up from the sensible heat of water at the skin surface. Consequently, the water temperature at the skin surface will drop even further. This process will continue until the latent heat of evaporation is equal to the rate of heat transfer to the water at the skin surface. Once steady conditions are obtained and the interface temperature stabilises, the energy balance on a thin layer of water at the skin can be expressed as follows: 


$$
\dot{q}_{e v}=\frac{\dot{m}_{h_{2} o} h_{f g}}{V_{s k}}
$$

where $\dot{m}_{h_{2} o}$ is the water evaporation flow rate, $h_{f g}$ is the latent heat of evaporation of water at the surface temperature and $V_{s k}$ is the total volume of the first cell layer adjustment to the skin from the body side.

The latent heat of evaporation can be obtained from the literature [14]. The calculation procedures performed by the $I H B M$ to obtain the evaporation rate $\dot{m}_{h_{2} \mathrm{o}}$ are discussed in detail in [13].

\subsection{Modelling respiration heat losses}

In certain situations, respiration heat losses can be very small in comparison to the remaining components of the total infant's heat balance, and their contribution has often been omitted in mathematical calculations. However, this term can be significant in cases of low air temperature and humidity. The $I H B M$ module developed in this study allows the inclusion of the respiration process in the total heat balance equations. This ability becomes especially important in exceptional and emergency situations in which the thermal properties of air make the respiration heat loss significant in the total heat balance.

In general, the convective and evaporative heat losses occurring during respiration can be calculated from the following equations [14]:

$$
\begin{aligned}
& \left(\dot{Q}_{c v}\right)_{l u}=\dot{m}_{a i r, l u} C_{p, a i r}\left(T_{c r}-T_{a m b}\right) \\
& \left(\dot{Q}_{e v}\right)_{l u}=\dot{m}_{a i r, l u}\left(w_{e x}-w_{a m b}\right) \cdot h_{f g}
\end{aligned}
$$

where $\dot{m}_{a i r, l u}$ is the rate of air intake to the lungs, $C_{p, a i r}$ is the specific heat of air, $T_{c r}$ is the deep body temperature, $T_{a m b}$ is the ambient temperature, $w_{e x}$ and $w_{a m b}$ are the humidity ratio in exhaled and ambient air, respectively, and $h_{f g}$ is the enthalpy of water evaporation. The total heat losses due to respiration are then given by

$$
\dot{q}_{l u}=\frac{\left(\dot{Q}_{c v}\right)_{l u}+\left(\dot{Q}_{e v}\right)_{l u}}{V_{\mathrm{inf}}}
$$

The $I H B M$ module allows the specification of the method for obtaining the ambient air properties. The first method allows the specification of the location within the domain from which the ambient parameters can be extracted, whereas the second option allows the calculation of the average value of required parameters based on the values obtained from each individual cell within the computational domain [13].

\section{CFD Simulations}

In general, most CFD applications follow the same basic methodology. Some of the differences include problem complexity, available computer resources, available CFD expertise, and whether a commercially available CFD package is used or a problem-specific algorithm is developed. The steps necessary to complete a CFD simulation can be classified into three main groups as follows: 
- Pre-processing, which involves activities necessary to build and set up the numerical model;

- Processing, involving the solution of the system of differential equations describing the problem under consideration;

- Post-processing, which involves the presentation of results in numerical or graphical form.

In this work, the validation of the CFD simulations is carried out by comparison with experimental measurements from the medical literature. The parametric capabilities of the proposed numerical techniques allowed adjusting the models to match some of the conditions during which the experiments took place.

The validation procedure was done gradually, by staging the introduction of the different components of the heat balance in the calculations. In the first stage, only dry heat losses from the neonate to the surrounding environment were investigated. These included metabolic heat generation and dissipation by conduction, convection and radiation. The next validation case also includes latent heat losses from the infant's skin and lungs. The final validation test demonstrates the ability of the CFD model to simulate the transient process of respiration.

\subsection{Dry heat losses}

The initial case analyses dry heat losses from a neonate within an incubator. Verification of the CFD simulations was performed by comparison with the results of Elabbassi et al. [19], who described experiments to assess the heat losses for small and large neonates using two thermal manikins, representing newborns of $900 \mathrm{~g}$ and $1800 \mathrm{~g}$. During the experiment, dry heat losses were measured from the six body segments of the manikins. The effect of different environmental thermal conditions was investigated with air temperatures ranging between 29 and $35^{\circ} \mathrm{C}$. As a result of the experiments, relationships between the total dry heat losses and mean air temperature for each manikin were established from linear regression as follows [19]:

$$
\begin{aligned}
& \left(\dot{Q}_{d r y}\right)_{l \text { arge }}=\left(490.03-12.16 T_{a m b}\right) A_{\mathrm{inf}} \\
& \left(\dot{Q}_{d r y}\right)_{\text {small }}=\left(745.70-19.25 T_{\text {amb }}\right) A_{\mathrm{inf}}
\end{aligned}
$$

where $\left(\dot{Q}_{d r y}\right)_{l \text { arge }}$ and $\left(\dot{Q}_{d r y}\right)_{\text {small }}$ represent the total dry heat loss from the large and the small manikin, respectively, $T_{a m b}$ represents the ambient temperature within the incubator, and $A_{\text {inf }}$ represents the infant's skin surface area.

The verification of the CFD results was based on the above relationships. Information in [19] allowed reconstruction of the geometry of the two manikins. Parametric capabilities of the model were used to match the size and position of the manikins. The IHBM module in conjunction with the FLUENT solver was used to perform the numerical calculations. However, since only dry heat losses from the infant's body were investigated in [19], the evaporation and respiration processes were not considered in the numerical simulations.

Due to the lack of technical documentation for the BioMS C 2750 incubator utilised in [19], the numerical simulations were performed based on a single-walled version of the Caleo $^{\mathrm{TM}}$ incubator, shown in Figures 1 and 2, manufactured by Dräger [20]. Hence, reasonable differences between the CFD and experimental results can be expected. In order to minimise these differences, the ambient flow field variables for the numerical calculations were set to match those measured during the experiments. Hence, an initial series of 
simulations was performed to establish the appropriate inlet air thermal properties capable of sustaining the ambient temperature for which the experiments were performed.

\subsubsection{Model geometry and discretization}

For complex CFD simulations involving multiple geometric parts created in different applications, the geometry and grid generation processes can be extremely challenging. In addition to the complexity of the configuration, some defects are usually present, such as discontinuous surfaces, cracks, and overlaps. Conventional geometry-based repair is very tedious and can consume large amounts of time. The surface wrapping technology available in the CFD pre-processor TGRID allowed merging two models previously created in different applications and produce a fully connected surface model. The technique is based on capturing a roughly defined volumetric region using a Cartesian grid. The grid is then intersected with the underlying geometry to form a shell, which is then projected back onto all faces on the geometry. Throughout the process, individual features from both models are selectively captured.

Geometric representations of the neonates were built by using $S T L$ models modified in $3 D$ STUDIO MAX to represent the manikins used in the experiments. Two different models were created to represent $900 \mathrm{~g}$ and $1800 \mathrm{~g}$ manikins. A typical model is shown in Figures 3. Utilization of $3 D$ STUDIO MAX combined with the wrapping technology available in the TGRID pre-processor proved to be very beneficial for generating complex and accurate models for the CFD simulations. It allowed using the pre-processor in a fully automatic way, thus substantially reducing the time required for completing this task.

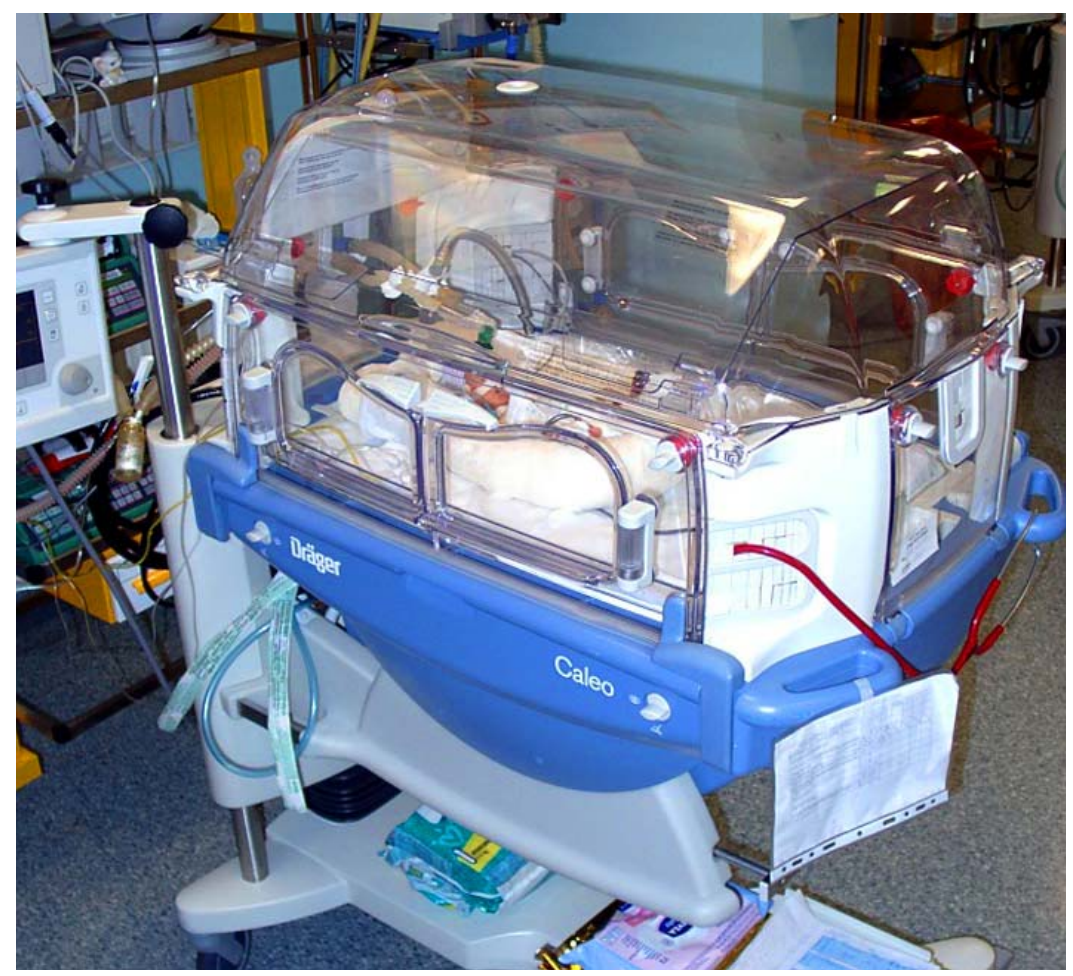

Figure 1. Caleo ${ }^{\mathrm{TM}}$ incubator manufactured by Dräger 


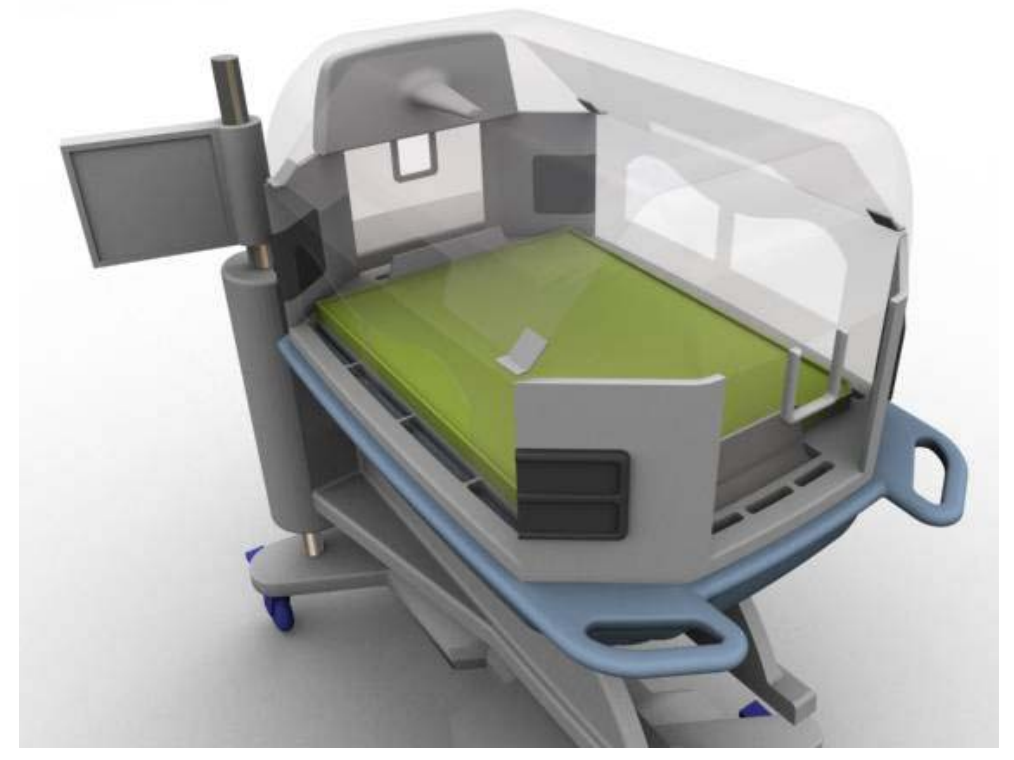

Figure 2. Three-dimensional model of the Caleo ${ }^{\mathrm{TM}}$ incubator generated using CATIA

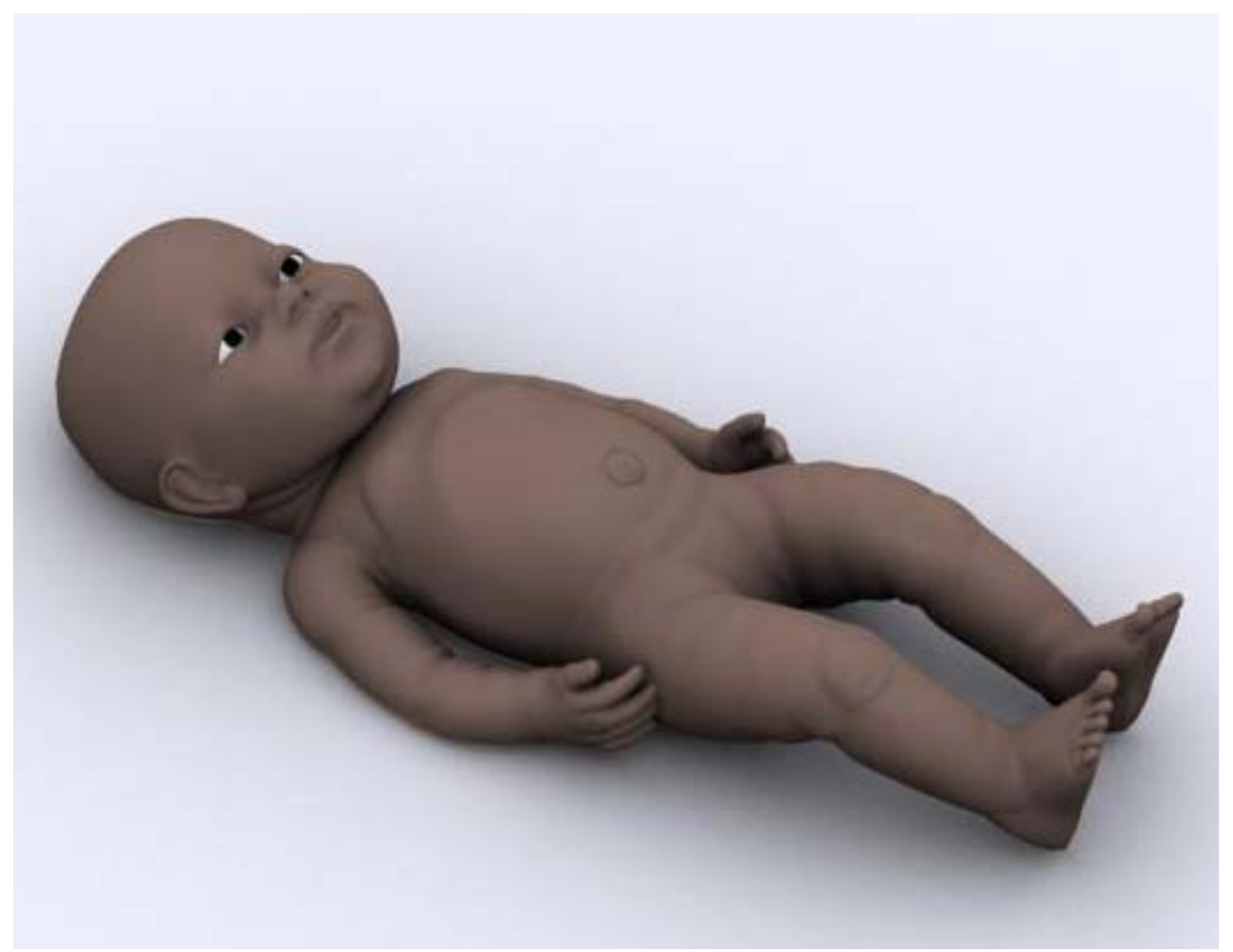

Figure 3. 3D Studio Max model of a neonate lying in relaxed supine position 


\subsubsection{Boundary conditions}

Velocity boundary conditions were used to define the constant inlet velocity and scalar properties of the flow at the inlet boundaries. However, the comparison of results was made based on the total dry heat losses from the infant's body expressed as a function of the ambient air temperature. Hence, additional simulations were performed in order to establish appropriate air inlet boundary conditions from which the required ambient air temperature could be obtained. Only the air temperature was set as a variable during those trials, and the air inlet velocity was assumed to be constant. The final results of the preliminary trials are presented in Table 1.

Table 1. Air inlet boundary conditions

\begin{tabular}{|c|c|c|c|c|c|c|c|}
\hline $\begin{array}{c}\text { Air temperature at } \\
\text { the incubator inlets } \\
\left({ }^{\circ} \mathrm{C}\right)\end{array}$ & 29.9 & 31.0 & 32.1 & 33.0 & 34.1 & 35.2 & 36.2 \\
\hline $\begin{array}{c}\text { Air ambient } \\
\text { temperature }\left({ }^{\circ} \mathrm{C}\right) \\
\text { CFD calculations }\end{array}$ & 29.1 & 29.9 & 31.0 & 31.9 & 33.1 & 34.1 & 35.1 \\
\hline $\begin{array}{c}\text { Air ambient } \\
\text { temperature }\left({ }^{\circ} \mathrm{C}\right) \\
\text { Elabbassi } \text { et al. }[19]\end{array}$ & 29.0 & 30.0 & 31.0 & 32.0 & 33.0 & 34.0 & 35.0 \\
\hline
\end{tabular}

The neonate was represented as a body in which heat is generated by metabolism and dissipated through the external skin layer. The amount of generated heat and its dissipation process were calculated by the $I H B M$ module based on the previous empirical formulas. Heat transfer inside the body is not taken into account in the simulations. The relevant information regarding the material properties of the body tissue and other required parameters was obtained from the literature $[6,11]$ and is presented in Table 2. Two different materials were used to model the opaque and semi-transparent walls of the incubator. The properties of those materials are presented in Tables 3 and 4 [14].

Table 2. Physical and physiological parameters of the infant's body

\begin{tabular}{|l|c|c|}
\hline & Small infant model & Large infant model \\
\hline Newborn's weight $(\mathrm{kg})$ & 0.900 & 1.800 \\
\hline Newborn's height $(\mathrm{m})$ & 0.38 & 0.51 \\
\hline Skin surface area $\left(\mathrm{m}^{2}\right)$ & 0.086 & 0.150 \\
\hline Age (days) & 1 & 1 \\
\hline Gestation (weeks) & 32 & 32 \\
\hline Tissue conductivity $\left(\mathrm{W} \mathrm{m}^{-1} \mathrm{~K}^{-1}\right)$ & \multicolumn{2}{|c|}{0.34} \\
\hline Tissue density $\left(\mathrm{kg} \mathrm{m}^{-3}\right)$ & \multicolumn{2}{|c|}{1000} \\
\hline Tissue specific heat $\left(\mathrm{J} \mathrm{kg}^{-1} \mathrm{~K}^{-1}\right)$ & \multicolumn{2}{|c|}{1000} \\
\hline Blood perfusion rate $\left(\mathrm{kg} \mathrm{m}^{-3} \mathrm{~s}^{-1}\right)$ & 3800 \\
\hline Blood density $\left(\mathrm{kg} \mathrm{m}^{-3}\right)$ & \multicolumn{2}{|c|}{} \\
\hline Blood specific heat $\left(\mathrm{J} \mathrm{kg}^{-1} \mathrm{~K}^{-1}\right)$ & \multicolumn{2}{|c|}{} \\
\hline
\end{tabular}


Table 3. External wall boundary conditions (opaque material)

\begin{tabular}{|c|c|c|}
\hline Material name & \multicolumn{2}{|c|}{ Plastic (PVC) } \\
\hline Total internal surface area & 1.10 & $\left(\mathrm{~m}^{2}\right)$ \\
\hline Total external surface area & 1.10 & $\left(\mathrm{~m}^{2}\right)$ \\
\hline Density & 1.700 & $\left(\mathrm{~kg} \mathrm{~m}^{-3}\right)$ \\
\hline Specific heat & 1400 & $\left(\mathrm{~J} \mathrm{~kg}^{-1} \mathrm{~K}^{-1}\right)$ \\
\hline Thermal conductivity & 0.14 & $\left(\mathrm{~W} \mathrm{~m}^{-1} \mathrm{~K}^{-1}\right)$ \\
\hline Absorption coefficient & 0 & $\left(\mathrm{~m}^{-1}\right)$ \\
\hline Internal emissivity & 0.90 & \\
\hline
\end{tabular}

Table 4. External wall boundary conditions (semi-transparent material)

\begin{tabular}{|c|c|c|}
\hline Material name & \multicolumn{2}{|c|}{ Glass fibre } \\
\hline Total internal surface area & 0.264 & $\left(\mathrm{~m}^{2}\right)$ \\
\hline Total external surface area & 0.266 & $\left(\mathrm{~m}^{2}\right)$ \\
\hline Density & 1450 & $\left(\mathrm{~kg} \mathrm{~m}^{-3}\right)$ \\
\hline Specific heat & 0.96 & $\left(\mathrm{~J} \mathrm{~kg}^{-1} \mathrm{~K}^{-1}\right)$ \\
\hline Thermal conductivity & 0.036 & $\left(\mathrm{~W} \mathrm{~m}^{-1} \mathrm{~K}^{-1}\right)$ \\
\hline Absorption coefficient & 220 & $\left(\mathrm{~m}^{-1}\right)$ \\
\hline Internal emissivity & 0.92 & \\
\hline
\end{tabular}

\subsubsection{Model discretisation and verification}

Several calculations with different mesh sizes have been performed to verify the accuracy of the simulations, as described in [12]. The number of points inside the incubator and in the exterior domain was set up in such a way that the values of air temperature and velocity for the different meshes could be compared. Moreover, quantities like heat transfer due to radiation and total heat transfer exchange between the infant and the surrounding environment were also monitored and compared. The meshes analysed in [12] were composed of tetrahedral elements, with typical converged meshes consisting of 1,500,000 elements.

The current simulations employed polyhedral meshes available in the new Fluent 6.4 Beta version. Polyhedral meshes allow a lower overall cell count compared to tetrahedral or hybrid meshes; typically, similar accuracy can be obtained with 3-5 times less elements for unstructured meshes. They also provide better convergence and stability during the calculations. A typical polyhedral mesh for the Caleo incubator is shown in Figure 4. 


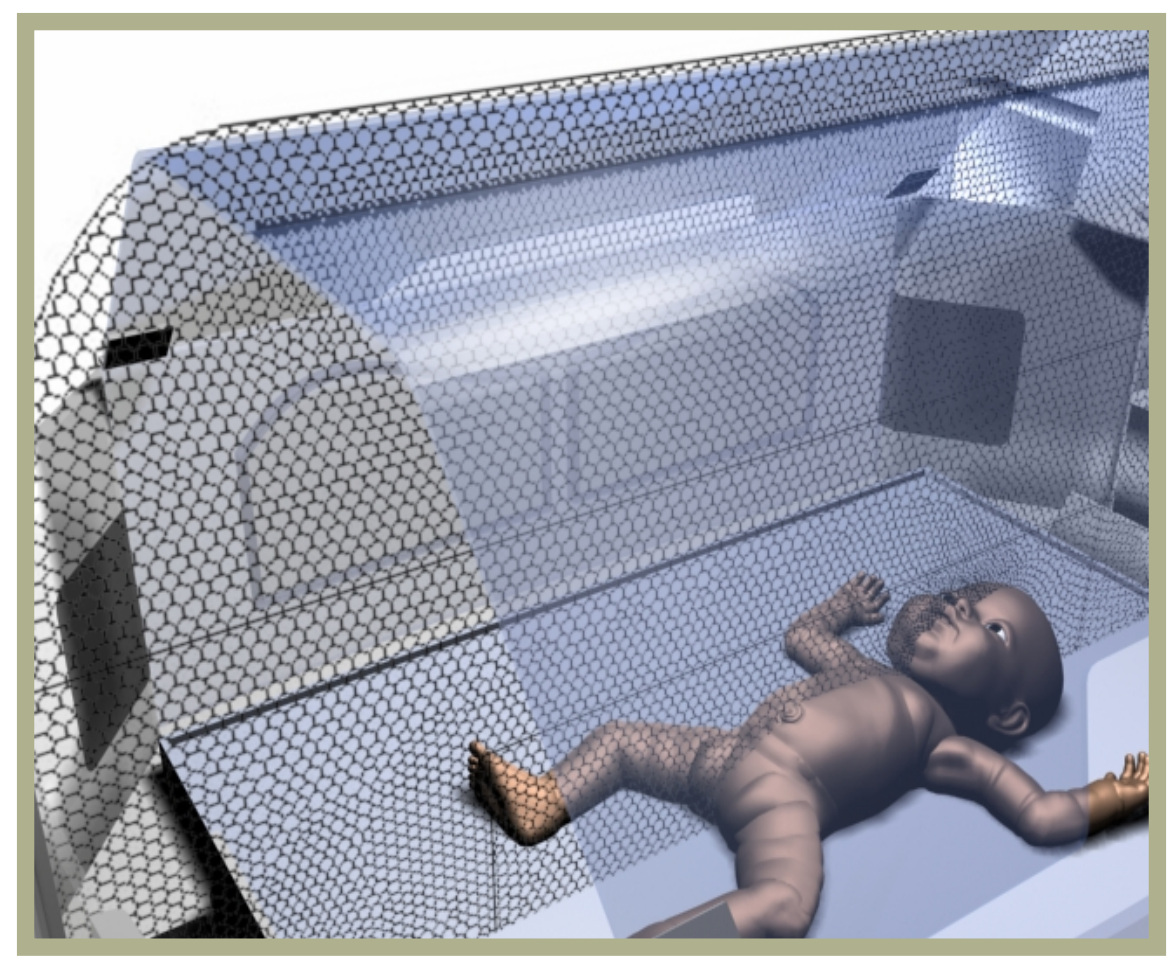

Figure 4. Polyhedral mesh for the Caleo ${ }^{\mathrm{TM}}$ incubator

\subsubsection{Results and discussion}

Simulations were performed for each model, representing two manikins of $900 \mathrm{~g}$ and $1800 \mathrm{~g}$. Seven different air ambient temperatures were investigated and compared with the results of Elabbassi et al. [19], as presented in Table 5.

The total dry heat losses obtained from the CFD simulations are approximately $20 \%$ lower than the experimental data of Elabbassi et al. [19]. Similar results were presented for a manikin of different size in [12] using a tetrahedral mesh. The comparison is qualitative rather than quantitative since different incubators were used in the CFD and experimental analyses. However, the results clearly indicate the correct trend with increased heat losses for the smaller manikin and a consistent $20 \%$ reduction in the total dry heat losses in all cases for the infants nursed in the more advanced $\mathrm{Caleo}^{\mathrm{TM}}$ incubator, which only confirms its increased efficiency over the much older BioMS C 2750 incubator used in [19]. Although the accuracy of the CFD simulation has been verified by mesh-independence tests, standard modelling and experimental errors also play a part in explaining the differences between the results.

Table 5. Comparison of total dry heat losses

\begin{tabular}{|c|c|c|c|c|}
\hline \multirow{2}{*}{$\begin{array}{c}\text { Ambient air } \\
\text { temperature within } \\
\text { the incubator } \\
\left({ }^{\circ} \mathrm{C}\right)\end{array}$} & \multicolumn{2}{|c|}{$\begin{array}{c}\text { Total dry heat loss from } \\
\text { large manikin } \\
\left(\mathrm{W} \mathrm{m}^{-2}\right)\end{array}$} & \multicolumn{2}{c|}{$\begin{array}{c}\text { Total dry heat loss from } \\
\text { small manikin } \\
\left(\mathrm{W} \mathrm{m}^{-2}\right)\end{array}$} \\
\cline { 2 - 5 } & $\begin{array}{c}\text { Elabbassi } \text { et al. } \\
{[19]}\end{array}$ & CFD & $\begin{array}{c}\text { Elabbassi } \text { et al. } \\
{[19]}\end{array}$ & CFD \\
\hline 29 & 137.4 & 108.3 & 187.5 & 169.1 \\
\hline 30 & 125.2 & 99.0 & 168.2 & 154.6 \\
\hline 31 & 113.1 & 89.5 & 149.0 & 134.5 \\
\hline 32 & 100.9 & 78.6 & 129.7 & 108.5 \\
\hline 33 & 88.7 & 70.5 & 110.5 & 92.1 \\
\hline 34 & 76.6 & 58.4 & 91.2 & 66.9 \\
\hline 35 & 64.4 & 46.9 & 72.0 & 44.3 \\
\hline
\end{tabular}




\subsection{Latent Heat Losses}

Wheldon and Hull [21] described a series of experiments investigating the metabolic heat generated by an infant's body and the evaporative heat losses from the infant's skin. Their results clearly demonstrated that, for premature infants with less than 30 weeks gestation, heat losses due to evaporation exceed $30 \%$ of the total metabolic heat generated in all analysed cases. Additionally, according to Agren et al. [22], the evaporation water losses from the infant's skin in certain situations can be as high as $60 \mathrm{~g} /\left(\mathrm{m}^{2} \mathrm{~h}\right)$. Such high evaporation rate would result in $3.6 \mathrm{~W}$ of latent heat loss caused only by the evaporation process for the $900 \mathrm{~g}$ infant analysed in the previous validation case. At the same time, the metabolic heat generated by the infant, based on the equation proposed by Brück [17], would only result in $1.5 \mathrm{~W}$. This particular example indicates the importance of evaporation heat losses in the total infant heat balance. In the case of term infants born after 32 gestation weeks, heat loss due to evaporation is less intensive. However, in lower humidity environments, it can still significantly influence the overall infant heat balance [23, 24].

Water loss from the infant's skin is only part of the total water losses due to evaporation. A similar process occurs within the lungs during respiration. The $I H B M$ module possesses the capability of calculating both the respiratory and transepidermal water losses from the infant's body.

A series of publications of Hammarlund et al [23, 24] also demonstrated that the evaporation heat losses from the infant's skin might be significantly higher than the dry heat losses, especially in the first hours of the infant's life. The evaporation of moisture from the infant's skin strongly influences the temperature profile and consequently, the total dry heat losses. Hence, experiments performed with manikins, as in the previous case, are limited in terms of the number of heat transfer modes that can be investigated. The analysis of evaporation and respiration heat losses with utilization of manikins is theoretically possible, but the costs of developing appropriate models would be substantially high. The CFD approach does not possess the same limitations and can take into the account all modes of heat transfer.

A series of validation trials has been performed based on extensive studies of Hammarlund et al. [23] and Sedin et al. [24], who provided detailed information on the weight, length, mass and gestation age for a group of 34 different infants analysed during their experimental studies. From this group, 19 infants were selected for the simulations, varying from 25 to 33 weeks gestation. More information on the test cases analysed and discussion of the results is given by Ginalski et al. [13].

\subsubsection{Results and discussion}

The validation of results has been performed with respect to experimental measurements available in the papers by Hammarlund et al. [23] and Sedin et al. [24]. Unfortunately, the articles did not include all the data required to reconstruct accurate representations of the infants analysed, and the properties of the incubator and the environment in which they were nursed. Hence, the comparison of the CFD results with data published in these articles can only provide limited validation of the CFD model.

The experiments of Hammarlund et al. [23] examined the evaporation rate from an infant's skin at different ambient air conditions. The latent heat loss was calculated as a product of evaporation rate and latent heat of evaporation of water. The infant selected for the CFD simulations was infant number 19 in [23], with the following characteristics: weight $1,900 \mathrm{~kg}$, length $0.44 \mathrm{~m}$, gestation age 33 weeks.

The mean skin temperature for the infant was calculated in the CFD simulations as a weighted average of each computational face representing the infant's skin. The body core 
temperature was calculated as the maximum temperature within the infant's body. The CFD results for the mean skin temperature and the body core temperature were $35.4^{\circ} \mathrm{C}$ and $36.8^{\circ} \mathrm{C}$, respectively, while the corresponding data obtained by Hammarlund et al. (1977) were $35.3 \pm 0.1^{\circ} \mathrm{C}$ and $36.3 \pm 0.1^{\circ} \mathrm{C}$.

CFD simulations were then performed for nine different ambient conditions, in which the air humidity ranged from 20 to $60 \%$. Air temperature and velocity are kept constant in all simulations. The results from the CFD simulations are compared to the experimental data in Table 6. It must be reiterated that different incubators were used for the simulation and the experiments, and also that there are several missing parameters in [23] related to the velocity field within the incubator and the external environment. Taking this into account, it can be concluded that the algorithm utilized by the IHBM module to calculate latent heat losses due to moisture evaporation from the infant's skin provides accurate results. Additionally, the Caleo $^{\mathrm{TM}}$ incubator [20] used in the CFD simulations is a more advanced neonatal unit than the AGA MK41 incubator used in the experiments, justifying the reduced heat losses. Again, standard modelling and experimental errors also play a part in the differences between the results.

Table 6. Comparison of evaporation heat loss results.

\begin{tabular}{ccc}
\hline $\begin{array}{c}\text { Relative air } \\
\text { humidity (\%) }\end{array}$ & $\begin{array}{c}\text { Evaporation heat loss (W) } \\
\text { Hammarlund et al. }[23]\end{array}$ & $\begin{array}{c}\text { Evaporation heat loss }(\mathrm{W}) \\
\text { CFD calculations }\end{array}$ \\
\hline 20 & 0.84 & 0.70 \\
25 & 0.79 & 0.66 \\
30 & 0.75 & 0.62 \\
35 & 0.70 & 0.58 \\
40 & 0.65 & 0.55 \\
45 & 0.61 & 0.50 \\
50 & 0.56 & 0.46 \\
55 & 0.51 & 0.43 \\
60 & 0.47 & 0.39 \\
\hline
\end{tabular}

The next validation test case concerns respiration heat losses. To validate this last remaining component of the infant heat balance, four different cases were investigated by making use of the full capabilities of the $I H B M$ module. The validation tests were performed on the basis of experimental data for respiratory heat losses obtained by Sulyok et al. [24], who analysed the influence of humidity and temperature on the insensible water loss from the infants using the direct calorimetric method. Information including infant weights, size and nursing position has been utilized as parameters to automatically generate representative infant models and is presented in Table 7, together with other parameters necessary for modelling the respiration process. Body tissue and blood properties are given in Table 2 .

Table 7. Physical characteristic of the infants.

\begin{tabular}{ccccccc}
\hline $\begin{array}{c}\text { Infant } \\
\text { number }\end{array}$ & $\begin{array}{c}\text { Weight } \\
(\mathrm{kg})\end{array}$ & $\begin{array}{c}\text { Length } \\
(\mathrm{m})\end{array}$ & $\begin{array}{c}\text { Tidal } \\
\text { lungs } \\
\text { volume } \\
(\mathrm{ml})\end{array}$ & $\begin{array}{c}\text { Lungs } \\
\text { dead } \\
\text { space } \\
(\mathrm{ml})\end{array}$ & $\begin{array}{c}\text { Respiration } \\
\text { rate } \\
(\mathrm{bth} / \mathrm{min})\end{array}$ & $\begin{array}{c}\text { Respiration } \\
\text { flow rate } \\
(\mathrm{ml} / \mathrm{min})\end{array}$ \\
\hline 1 & 3.242 & 0.50 & 22.7 & 8.1 & 52 & 379.6 \\
2 & 3.207 & 0.49 & 22.4 & 8.0 & 48 & 345.6 \\
3 & 3.277 & 0.52 & 22.9 & 8.2 & 50 & 367.5 \\
4 & 3.196 & 0.51 & 22.4 & 8.0 & 57 & 410.1 \\
\hline
\end{tabular}


In the first stage, deep body and skin temperatures have been compared with the corresponding experimental data [24], as presented in Table 8. The maximum deep body temperature difference between the CFD and experimental results was less than $0.6^{\circ} \mathrm{C}$, while the maximum difference for the average skin temperature was less than $0.8^{\circ} \mathrm{C}$. These results indicate the good accuracy of the CFD calculations, in line with the previous cases. The difference between the experimental and numerical results can be justified by the lack of some of the data necessary to fully describe the model in [24].

Table 8. Comparison of body temperature results.

\begin{tabular}{ccccc}
\hline \multirow{2}{*}{$\begin{array}{c}\text { Infant } \\
\text { number }\end{array}$} & \multicolumn{2}{c}{ Sulyok et al. [24] } & \multicolumn{2}{c}{ CFD calculations } \\
\cline { 2 - 5 } & $\begin{array}{c}\text { Mean skin } \\
\text { temperature } \\
\left({ }^{\circ} \mathrm{C}\right)\end{array}$ & $\begin{array}{c}\text { Body core } \\
\text { temperature } \\
\left({ }^{\circ} \mathrm{C}\right)\end{array}$ & $\begin{array}{c}\text { Mean skin } \\
\text { temperature } \\
\left({ }^{\circ} \mathrm{C}\right)\end{array}$ & $\begin{array}{c}\text { Body core } \\
\text { temperature } \\
\left({ }^{\circ} \mathrm{C}\right)\end{array}$ \\
\hline 1 & 35.4 & 36.7 & 35.3 & 37.3 \\
2 & 36.0 & 36.9 & 36.7 & 37.4 \\
3 & 35.7 & 36.7 & 34.9 & 37.0 \\
4 & 35.5 & 36.6 & 35.9 & 36.9 \\
\hline
\end{tabular}

The average contribution of the respiration heat losses within the total heat losses caused by moisture evaporation from the respiratory tract and from the infant's skin is $42 \%$ for the experimental results and $45 \%$ for the CFD results. These numbers indicate the importance of properly modelling respiration heat losses.

\subsection{Transient modelling of the respiration process}

The ability to model respiration as a transient process is very important in some situations that include oxygen concentration studies and drug delivery. For this reason, a series of simulations has been performed in order to investigate the distribution of oxygen concentration under an oxygen hood. This particular device is used in neonatology to increase the oxygen concentration in the air provided to the neonate, to intensify metabolic processes. The main objective of this analysis was to fully simulate the transient air flow under the oxygen hood. The relatively small dimensions of this device enhanced the importance of the respiration process on the overall air flow pattern surrounding the infant. However, it must be stressed that the numerical simulations have not been validated and the manufacturer only provided a brief description of the model. The main purpose of the simulation was to demonstrate the full potential of the CFD technique in modelling complex heat and mass transfer processes in neonatology.

The computational domain for the calculations included only the head of the infant placed under the oxygen hood. The geometrical model can be seen in Figure 6. The hood geometry was created based on information from the manufacturer.

The quality of the mesh was, once again, verified by mesh-independence tests which focused on node point distribution, smoothness and cell shape. As usual, the mesh was more refined near the salient features of the flow (such as boundary layers, separated regions, shear layers). Rapid changes in the cell volume between adjacent cells were avoided to minimise truncation errors. The aspect ratio of the cells was kept at less than 5:1, and their skewness was also controlled to avoid the appearance of degenerated cells. The final computational domain is presented in Figure 7, and the final mesh had a total of 1,320,000 polyhedral elements. 


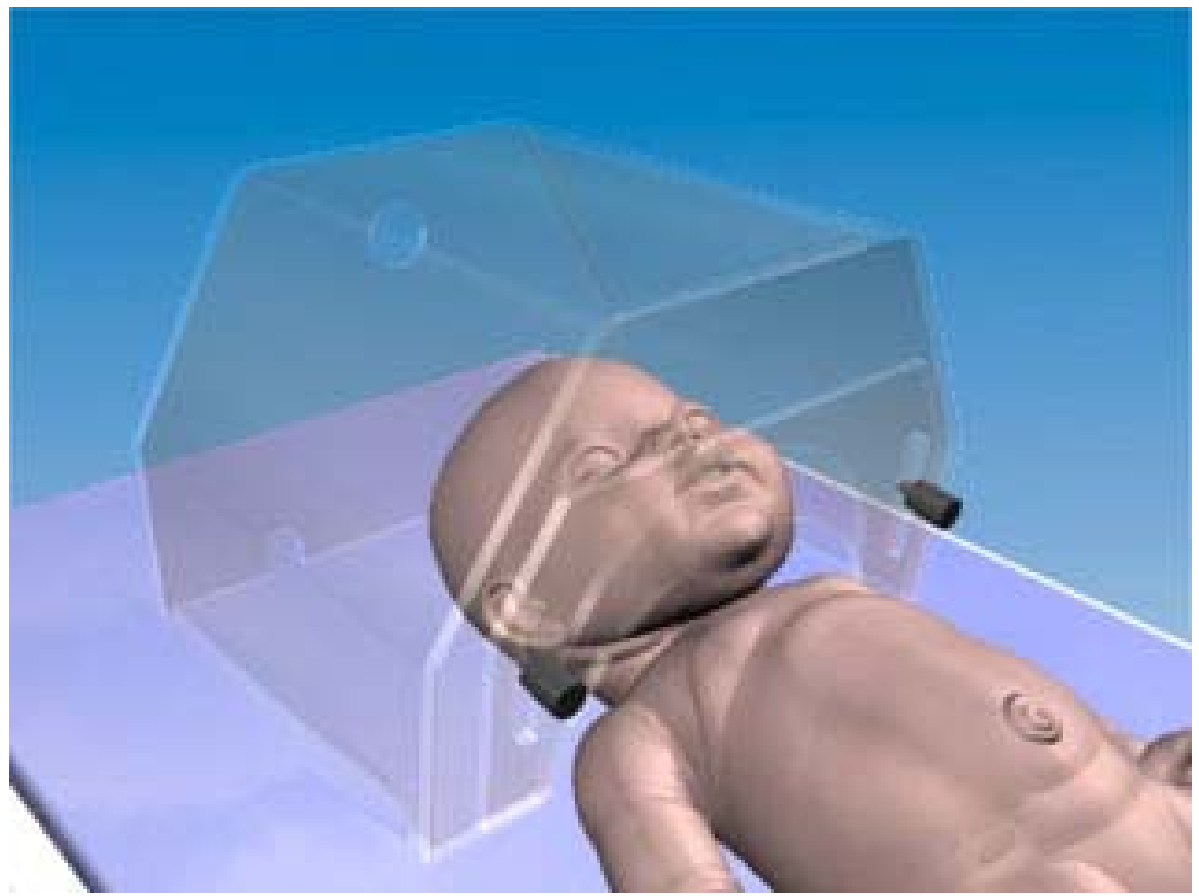

Figure 6. Geometrical model of a neonate nursed under oxygen hood.

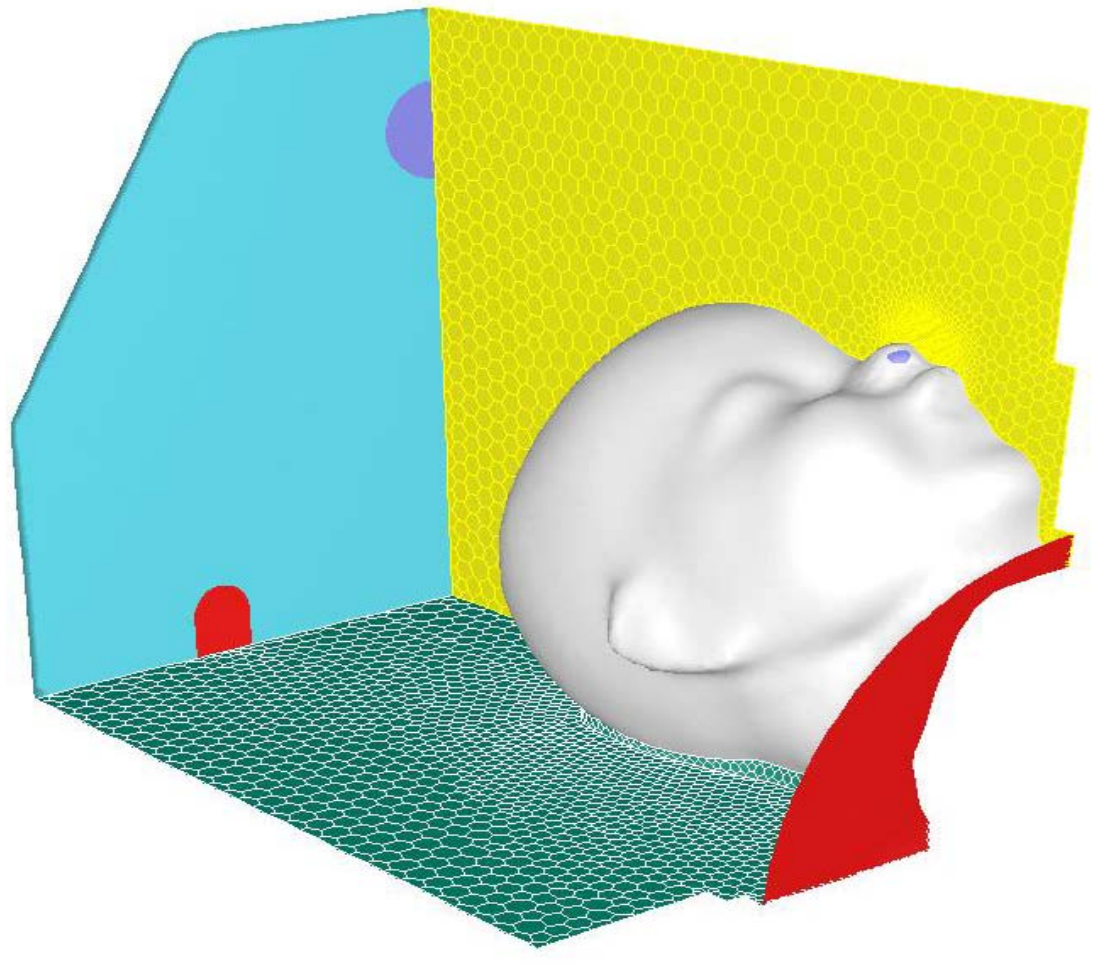

Figure 7. Model of the neonate's head placed under the oxygen hood, meshed with polyhedral elements

The $k-\varepsilon$ turbulence model was utilized in the calculations due to the high Reynolds numbers at the exhaled stage of breathing. All calculations were performed for the same infant characteristics as in the previous validation case regarding respiration heat losses. In order to limit the number of cells in the simulation, the centre line was considered as an axis of symmetry. Air was considered to be a multi-species mixture of oxygen, nitrogen, carbon 
dioxide and water vapour. An incompressible ideal gas law was employed to take into account density variations caused by temperature differences. The standard capabilities of the FLUENT solver were used in the simulation of this transient process.

Velocity boundary conditions were set at the inlets to the computational domain and at the infant nostrils. The respiration pattern was defined by the following function [6]:

$$
u_{\text {nos }}=\frac{\eta\left(V_{\text {tid }}-V_{\text {ded }}\right)}{\rho_{\text {air }, e x} A_{\text {nos }}} \sin (2 \pi t)
$$

where $u_{\text {nos }}$ represents velocity magnitude at the nostril; $\eta$ represents the respiration rate; $V_{\text {tid }}$ represents the lungs tidal volume; $V_{\text {ded }}$ represents the lungs dead space; $\rho_{\text {air }, \text { ex }}$ represents the density of the exhaled air; and $A_{n o s}$ represents the nostrils surface area. The information required to establish the velocity boundary condition at the infant nostrils is provided in Table 9 [11].

Table 9. Physical characteristic of the neonate

\begin{tabular}{lcc}
\hline Lungs tidal volume & $2.27 \times 10^{-4}$ & $\left(\mathrm{~m}^{3}\right)$ \\
Lungs dead space & $8.1 \times 10^{-5}$ & $\left(\mathrm{~m}^{3}\right)$ \\
Respiratory rate & 52 & $\left(\mathrm{~s}^{-1}\right)$ \\
\hline
\end{tabular}

The numerical simulation was performed for a time interval of 25 minutes of regular breathing, and aimed at demonstrating the potential capabilities of CFD techniques for transient modelling of the respiration process. Hence, the results obtained at this point have not been validated. However, several important conclusions can be obtained. For example, the oxygen concentrations in the air provided to the oxygen hood and in the air inhaled by the infant are considerably different. The respiration pattern and other related parameters will also influence the oxygen concentration in the inhaled air. CFD techniques can help to determine the level of oxygen concentration and the optimum position of the infant's head under the hood. Figure 8 shows a sequence of snapshots of the oxygen mole fraction during the expiration stage. The snapshots have been taken at the $25^{\text {th }}$ minute of the simulation. 

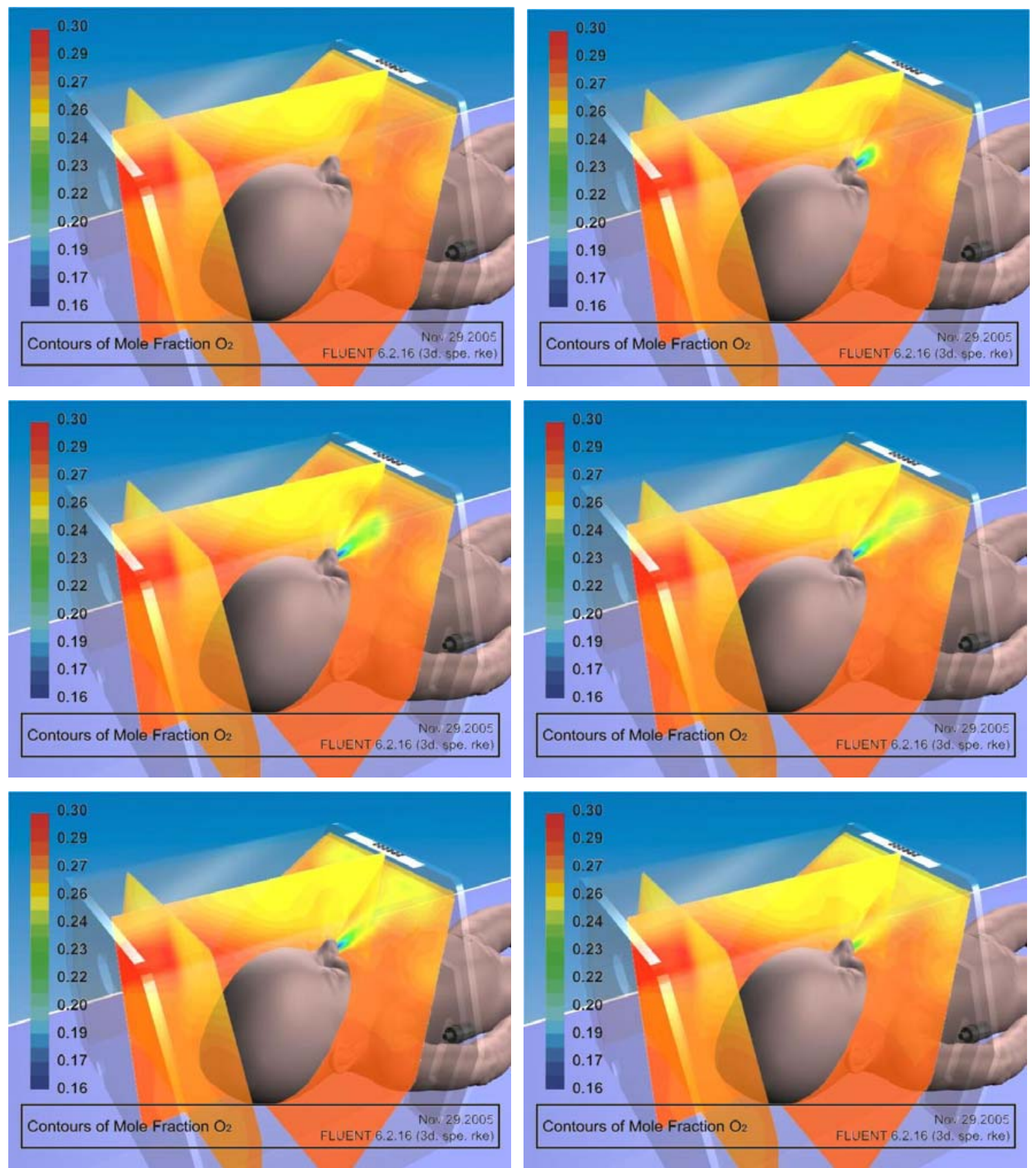

Figure 8. Contours of oxygen mole fraction plotted at different stages of the respiration cycle

The distribution of carbon dioxide under the hood during the respiration process can be seen in Figure 9. In comparison to oxygen, carbon dioxide dissipates quickly within the domain. Hence, the concentration profile of this gas is much more homogeneous confirming that the oxygen hood is properly ventilated. 

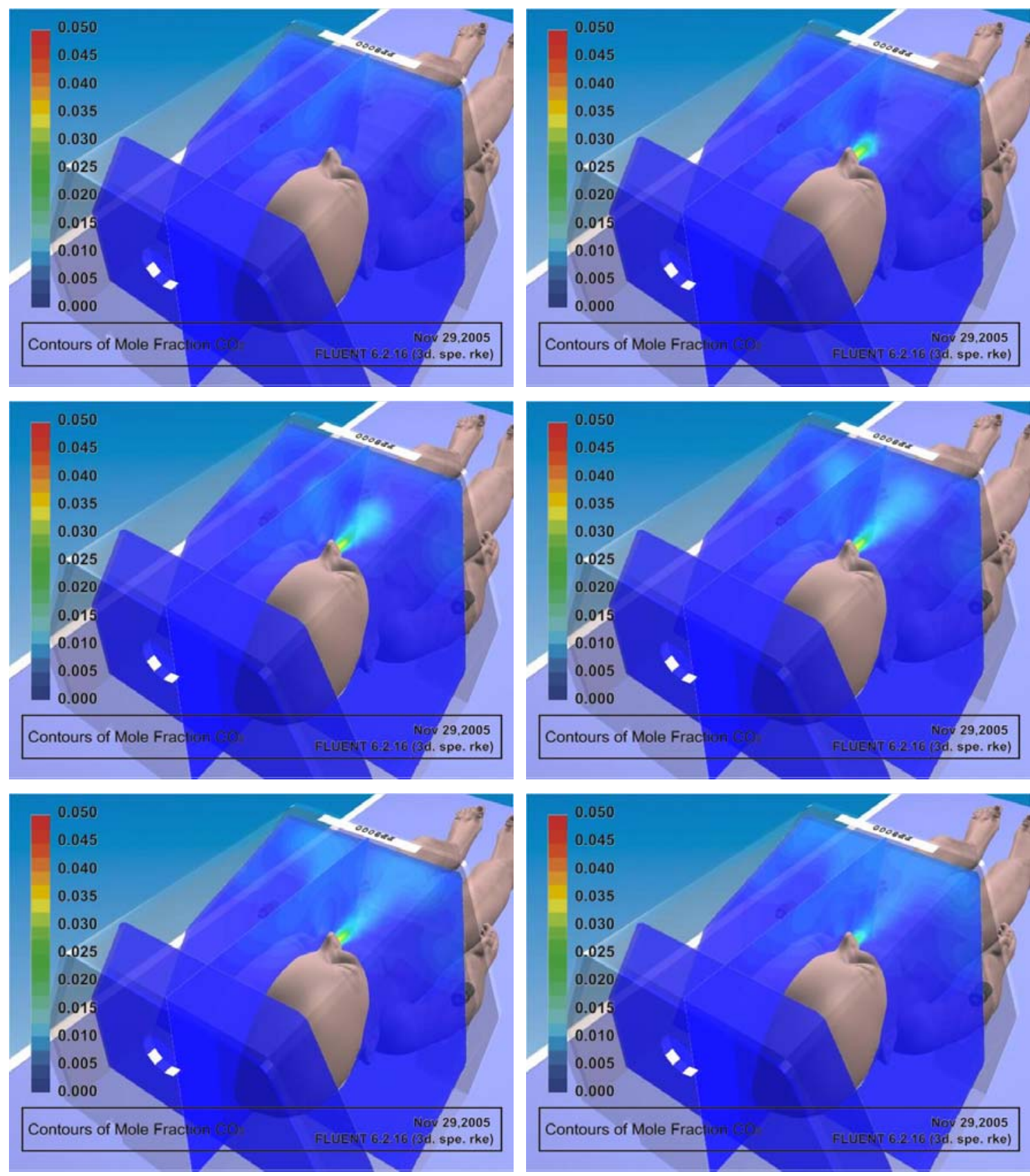

Figure 9. Contours of carbon dioxide mole fraction plotted at different stages of the respiration cycle

\section{Conclusions}

This paper presented CFD studies of the heat balance of neonates nursed within an incubator or an oxygen hood. The CFD simulations have been validated against experimental measurements from the medical literature, and proved to be reasonably accurate in estimating the core and skin temperatures of infants nursed within incubators. The study also demonstrated the CFD capabilities of simulating transient processes occurring within oxygen hoods.

The studies carried out in this paper aim at optimising the design of medical devices used in neonatology, in order to improve their performance. A recently started research investigates design improvements to radiant warmers, which are used to nurse unstable 
neonates requiring continuous interventions. As the infants are nursed in an open environment, a large amount of radiant heat is lost to the surroundings. Furthermore, as the neonates may be observed under a radiant warmer for several hours, water losses may be up to 50\% higher than in incubators, increasing the risk of dehydration [25]. The usual remedy to decrease water loss is to wrap the infant in a plastic shield, but this impairs the access to the neonates. It is expected that CFD simulations may provide a better understanding of the problem, leading to better procedures for the management of radiant warmers.

A further study under consideration will aim at helping determining whether the use of body cooling following perinatal asphyxia is a safe treatment that will improve survival and reduce neurological and neurodevelopmental impairments. These computer trials will test the feasibility of brain cooling using different types of cooling mattresses, and have the potential to reduce the number of clinical trials normally required for this type of study.

\section{References}

[1] Murakami S, Zeng J and Hayashi T 1999 CFD analysis of wind environment around a human body Journal of Wind Engineering and Industrial Aerodynamics 83 393-408

[2] Murakami S, Kato S and Zeng J 2000 Combined simulation of airflow, radiation and moisture transport for heat release from a human body Building and Environment 35 489-500

[3] Makinen T T, Gavhed D, Holmer I and Rintamaki H 2001 Effect of metabolic rate on thermal responses at different air velocities in $-10^{\circ} \mathrm{C}$ Comparative Biochemistry and Physiology A - Molecular and Integrative Physiology 128 759-68

[4] Sorensen D N and Voigt L K 2003 Modelling flow and heat transfer around a seated human body by computational fluid dynamics Building and Environment 38 753-62

[5] Strand F L 1980 Physiology - A Regulatory System Approach (London: Collier Macmillan)

[6] Guyton A C and Hall J E 2000 Textbook of Medical Physiology (Philadelphia: W B Saunders)

[7] Bach V, Telliez F, Krim G and Libert J P 1996 Body temperature regulation in the newborn infant: Interaction with sleep and clinical implications Neurophysiologie Clinique $\mathbf{2 6}$ 379-402

[8] Wasner J 1994 Heat Balance of Premature Infants (Lubeck,: Dragwerk AG)

[9] Bolton D P G, Nelson E A S, Taylor B J and Weatherall I L 1996 Thermal balance in infants Journal of Applied Physiology 80, 2234-42

[10] Wheldon A E and Rutter N 1982 The heat balance of small babies nursed in incubators and under radiant warmers Early Human Development 6 131-43

[11] Rennie J M and Robetron N R C 2002 A Manual of Neonatal Intensive Care (New York: Oxford University Press)

[12] Ginalski M K, Nowak A J and Wrobel L C 2007 A combined study of heat and mass transfer in a double-walled infant incubator Medical Engineering and Physics 29 531-41

[13] Ginalski M K 2007 Numerical analysis of heat and mass transfer processes within an infant incubator. PhD Thesis, Silesian University of Technology, Gliwice, Poland.

[14] Cengel Y 2003 Heat Transfer. A Practical Approach (New York: McGraw-Hill)

[15] Fluent 6.3 User Manual 2006 (Lebanon, NH: Fluent Inc)

[16] Wheldon A E 1982 Energy balance in the newborn baby: use of a manikin to estimate radiant and convective heat loss Physical \& Medical Biology 27 285-296

[17] Rubinsky B 2006 Numerical bioheat transfer, Chapter 26 in Handbook of Numerical Heat Transfer (Hoboken, NJ: Wiley) 
[18] Brück K 1961 Temperature regulation in the newborn Biology of the Neonate 3 65-119

[19] Elabbassi E B, Belghazi K, Delanaud S and Libert J P 2004 Dry heat loss in incubator: comparison of two premature newborn sized manikins European Journal of Applied Physiology 92 679-82

[20] Caleo Neonatal Incubator. Instructions for Use 2002 (Lübeck, Germany: Dräger Medical AG \& Co. KGaA)

[21] Wheldon A E and Hull D 1985 Incubation of very immature infants Arch Dis Child. 58 504-8

[22] Agren J, Sjors G and Sedin G 1998 Transepidermal water loss in infants born at 24 and 25 weeks of gestation Acta Paediatrica 87 1185-90

[23] Hammarlund K, Nilsson G E, Oberg P A and Sedin G 1977. Transepidermal water loss in newborn infants. I. Relation to ambient humidity and site of measurement and estimation of total transepidermal water loss Acta Paediatrica Scand. 66 553-62

[24] Sedin G, Hammarlund K and Stromberg B 1983 Transepidermal water loss in full-term and pre-term infants Acta Paediatrica Scand. (Supplement) 31 305-27

[25] Merenstein G B and Gardner S L 2006 Handbook of Neonatal Intensive Care (St Louis: Mosby) 